\title{
A contribution to the knowledge of Agromyzidae (Diptera) of Zlaté Hory and its vicinity (Czech Republic)
}

A contribution to the knowledge of Agromyzidae (Diptera) of Zlaté Hory and its vicinity (Czech Republic). - Acta Mus. Siles. Sci. Natur., 70: 183-192, 2021.

\begin{abstract}
Results of a biodiversity investigation of Agromyzidae (Diptera) in the cadastre of Zlaté Hory town (Silesia, Czech Republic) in June 2017 are presented. In the area under study a total of 43 species of 12 genera have been recorded including 29 species representing new additions to the fauna of Czech Silesia. Agromyza seticercus, Cerodontha (Poemyza) beigerae, Galiomyza galiivora, Liriomyza europaea, L. puella, Phytomyza aurei, Ph. cirsii, Ph. vilnensis and Pseudonapomyza palliditarsis being to most interesting species found in the area. The importance of these species from a faunistic point of view is discussed.
\end{abstract}

Keywords. Diptera, Agromyzidae, faunistics, Zlaté Hory, Silesia, Czech Republic

\section{Introduction}

The Agromyzidae belongs to the most species-diverse but relatively well studied families of Diptera in the Czech Republic: a total of 495 species are currently known from the country, with 408 species recorded from Moravia. In spite of this, the biodiversity and distribution of species of this interesting family are very poorly known in Silesia (representing northernmost part of Moravian region) and, particularly, in the vicinity of the Zlaté Hory town. First records of Agromyzidae in the territory of Silesia were published by B. Starý (1930) and by Zavřel (1953) who largely presented records of larval mines on host plants only, but without documentary material of reared or captured adults from the localities monitored. Several species of agromyzid flies from localities in Czech Silesia were also reported by Nowakowski (1973) in his monograph of the European species of the genus Cerodontha. Subsequently, Vála \& Roháček (1983) recorded 21 species of Agromyzidae from the raised peat-bog at Rejvíz village (lying within the Zlaté Hory cadastre) collected in the framework of the research project "Acalyptrate Diptera of peat-bogs of Northen Moravia". Most recently, Černý et al. (2021) reported Phytomyza vilnensis Pakalniškis, 1998 from Zlaté Hory as first record from the Czech Republic. In this contribution results of a faunistic research of mining flies in the vicinity of Zlaté Hory are presented, including a number of new records for the Silesian fauna.

\section{Material and methods}

All material examined was collected by means of sweeping (using a light net) on various types of low vegetation during 3 days in June 2017 at 18 localities in the cadastral territory of Zlate Hory town (Figs 1-7). All documented material (adult flies) is deposited in the collection of author. The faunistically interesting records are commented on. Taxa (genera and species) are arranged alphabetically in two subfamilies, Agromyzinae and Phytomyzinae.

Abbreviations used in text: CHKO - Protected Landscape Area (PLA), det. - determined, coll. - collection, NP - National Park, NPR - National Natural Reserve, PP - Natural Monument, PR - Natural Reserve, Res. reserve.

Nomenclature and distribution are based on the Fauna Europaea (Martinez 2013) and also follows Papp \& Černý $(2015,2016,2017,2020)$ and Spencer (1990). 


\section{Results}

\section{A LIST OF SPECIES}

\section{SUBFAMILY: AGROMYZINAE}

\section{Agromyza conjuncta Spencer, 1966}

Material examined: Zlaté Hory $0.5 \mathrm{~km} \mathrm{~N}$, PP Zlaté jezero (Res.), 50 $16^{\prime} 40^{\prime \prime} \mathrm{N}, 17^{\circ} 23^{\prime} 56^{\prime \prime E}, 380 \mathrm{~m}$, vegetation near bank of pond, 1 ふै, 21.vi.2017.

Agromyza mobilis Meigen, 1830

Material examined: Rejvíz, 50 $13^{\circ} 16^{\prime \prime} \mathrm{N}, 17^{\circ} 17^{\prime} 37^{\prime \prime} \mathrm{E}, 770 \mathrm{~m}$, Nature trail Rejvíz, forest road vegetation, 1 ,

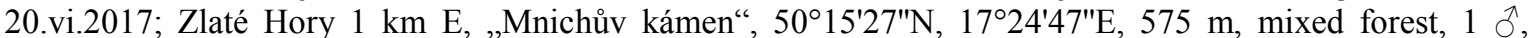
19.vi.2017; Zlaté Hory $1.5 \mathrm{~km} \mathrm{SW}$, „Zlatorudné mlýny“, $50^{\circ} 15^{\prime} 02^{\prime \prime} \mathrm{N}, 17^{\circ} 21^{\prime} 50^{\prime \prime} \mathrm{E}, 460 \mathrm{~m}$, near stream

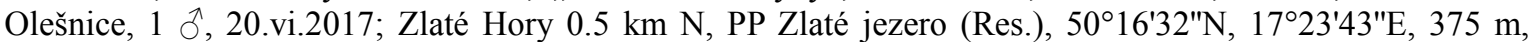
meadow near stream Zlatý potok, 1 ก , 21.vi.2017.

Agromyza nigripes Meigen, 1830

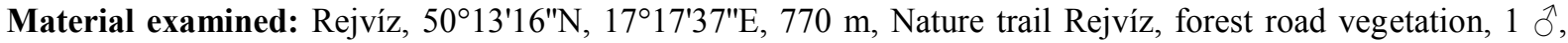

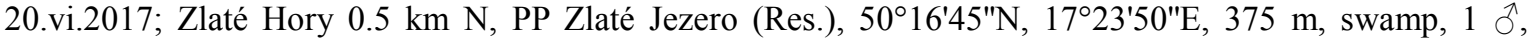

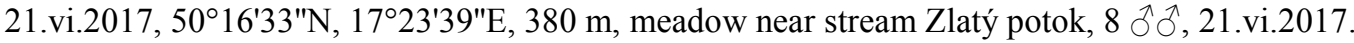

Agromyza seticercus L. Papp in Papp \& Černý 2015

Material examined: Rejvíz, $50^{\circ} 13^{\prime} 26^{\prime \prime} \mathrm{N}, 17^{\circ} 17^{\prime} 59^{\prime \prime} \mathrm{E}, 760 \mathrm{~m}$, Nature trail Rejvíz, margin of meadow, 2 ○े, 20.vi.2017; Zlaté Hory $0.5 \mathrm{~km} \mathrm{~N}$, PP Zlaté jezero (Res.), 50¹6'40"N, 17²3'56"E, $380 \mathrm{~m}$, bank of pond, 1 \%, 21.vi.2017.

Remarks: A species of Palaearctic distribution, originally described from Hungary (Papp \& Černý 2015) and subsequently confirmed from Ukraine (Guglya 2016) and then also found in other European countries (Croatia, Czech Republic, Netherlands, Slovakia and Switzerland - see Černý 2018, Černý \& Bächli 2018, Černý \& Roháček 2020, von Tschirnhaus \& van Wielink 2020) and also in Uzbekistan (Černý 2018). In the Czech Republic it has been recorded from several localities in Bohemia and southern Moravia (Černý 2018) but unknown from Northern Moravia. The above records are the first from Northern Moravia and Czech Silesia.

Melanagromyza aenea (Meigen, 1830)

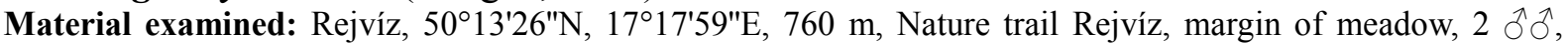
20.vi.2017.

Melanagromyza sativae Spencer, 1957

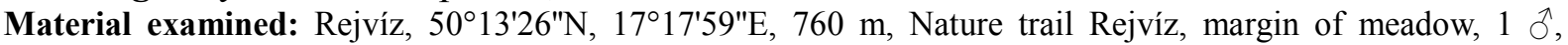
20.vi.2017.

Ophiomyia orbiculata (Hendel, 1931)

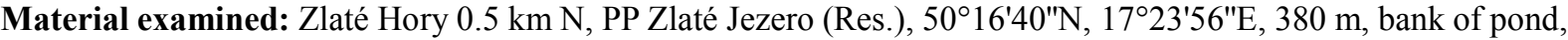

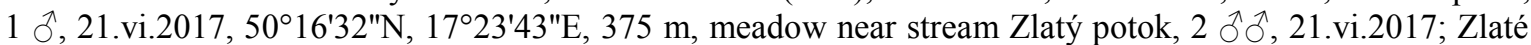
Hory $1.5 \mathrm{~km} \mathrm{~S}$, „Zlatorudné mlýny“, 50¹5'02"N, 17²1'50"E, $460 \mathrm{~m}$, near stream Olešnice, 1 ô, 20.vi.2017.

Ophiomyia pulicaria (Meigen, 1830)

Material examined: Zlaté Hory $1.5 \mathrm{~km} \mathrm{~S}$, „Zlatorudné mlýny“, $50^{\circ} 15^{\prime} 02^{\prime \prime} \mathrm{N}, 17^{\circ} 21^{\prime} 50^{\prime \prime} \mathrm{E}, 460 \mathrm{~m}$, near stream Olešnice, 1 ô, 20.vi.2017.

\section{SUBFAMILY: PHYTOMYZINAE}

Amauromyza (Cephalomyza) monfalconensis (Strobl, 1909)

Material examined: Zlaté Hory $0.5 \mathrm{~km}$ N, PP Zlaté Jezero (Res.), 50 $0^{\circ} 16^{\prime} 33^{\prime \prime} \mathrm{N}, 17^{\circ} 23^{\prime} 39^{\prime \prime} \mathrm{E}, 380 \mathrm{~m}$, meadow near

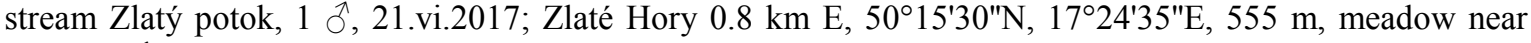
forest, $1 \hat{\jmath}, 19 . v i .2017$.

Cerodontha (Dizygomyza) bimaculata (Meigen, 1830)

Material examined: Rejvíz, „U pomníku“, $50^{\circ} 13^{\prime} 35^{\prime \prime N}, 17^{\circ} 17^{\prime} 46^{\prime} \mathrm{E}, 800 \mathrm{~m}$, forest road vegetation, 1 万ै, 20.vi.2017.

Cerodontha (Icteromyza) capitata (Zetterstedt, 1848)

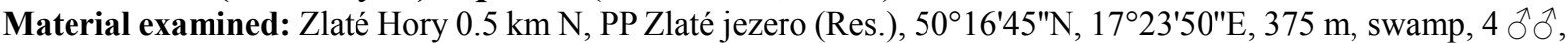
21.vi.2017, $50^{\circ} 16^{\prime} 40^{\prime \prime} \mathrm{N}, 17^{\circ} 23^{\prime} 56^{\prime \prime} \mathrm{E}, 380 \mathrm{~m}$, bank of pond, 23 ते oे $^{2} 21 . v i .2017$. 
Cerodontha (Poemyza) atra (Meigen, 1830)

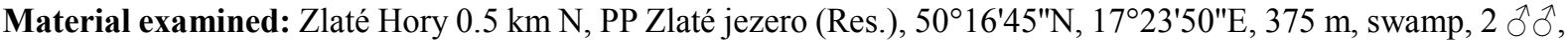

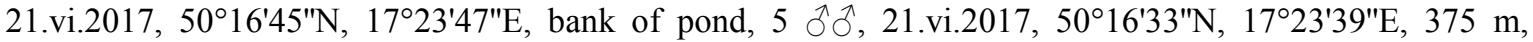

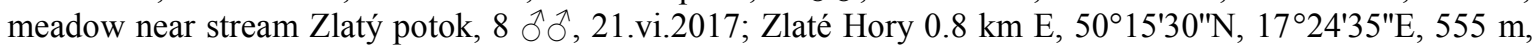
meadow near forest, $11 \hat{\jmath} \hat{\partial}^{2}, 19 . v i .2017$.

Cerodontha (Poemyza) beigerae Nowakowski, 1973

Material examined: Rejvíz, Nature trail Rejvíz, $50^{\circ} 13^{\prime} 25^{\prime \prime} \mathrm{N}, 17^{\circ} 17^{\prime} 13^{\prime \prime} \mathrm{E}, 798 \mathrm{~m}$, forest road vegetation, 1 ○े, 20.vi.2017.

Remarks: A Palaearctic species, in Europe known from the Czech Republic, Germany, Hungary, Poland, Sweden and Switzerland (Papp \& Černý 2016). In the Czech Republic it has only been reported from Bohemia from Vysoká nad Labem (Černý \& Vála 2005) and a few localities from CHKO Brdy (Heřman et al. 2019). Recently, it has also been confirmed in Moravia from Jihlava-Pávov (Černý 2018). The above record is the first from the Northern Moravia and Silesia.

Cerodontha (Poemyza) incisa (Meigen, 1830)

Material examined: Zlaté Hory $0.5 \mathrm{~km} \mathrm{E}, 50^{\circ} 15^{\prime} 30^{\prime \prime} \mathrm{N}, 17^{\circ} 24^{\prime} 11^{\prime \prime} \mathrm{E}, 520 \mathrm{~m}$, forest underbrush near forest road, 1 ô, 19.vi.2017.

Cerodontha (Poemyza) lateralis (Macquart, 1835)

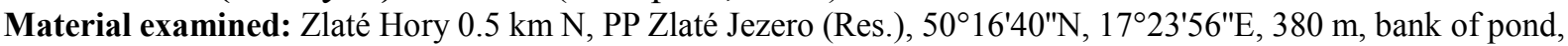
$1 \partial^{\lambda}, 21 . v i .2017,50^{\circ} 16^{\prime} 33^{\prime \prime} \mathrm{N}, 17^{\circ} 23^{\prime} 39^{\prime \prime E}, 380 \mathrm{~m}$, meadow near stream Zlatý potok, 1 đ̊, 21.vi.2017.

Cerodontha (Poemyza) muscina (Meigen, 1830)

Material examined: Rejvíz, „U pomníku“, $50^{\circ} 13^{\prime} 35^{\prime \prime N}, 17^{\circ} 17^{\prime} 46^{\prime} \mathrm{E}, 800 \mathrm{~m}$, forest road vegetation, 1 \%, 20.vi.2017.

Cerodontha (Poemyza) pygmella (Hendel, 1931)

Material examined: Zlaté Hory $2 \mathrm{~km}$ E, „Biskupská kupa“, 50¹5'24"N, 17²4'43"E, 890 m, vegetation near view-tower, 3 ठै, 19.vi.2017.

Cerodontha (Xenophytomyza) biseta (Hendel, 1920)

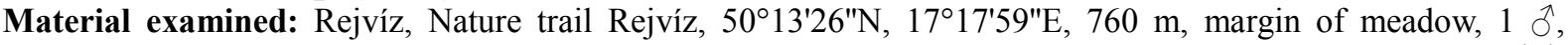
20.vi.2017; Zlaté Hory $0.5 \mathrm{~km} \mathrm{E,} 50^{\circ} 15^{\prime} 32^{\prime \prime} \mathrm{N}, 17^{\circ} 24^{\prime} 16^{\prime \prime} \mathrm{E}, 530 \mathrm{~m}$, forest underbrush near forest road, 4 o $^{\wedge}{ }^{7}$, 19.vi.2017; Zlaté Hory $1.2 \mathrm{~km} \mathrm{E}, 50^{\circ} 15^{\prime} 28^{\prime \prime} \mathrm{N}, 17^{\circ} 24^{\prime} 55^{\prime \prime} \mathrm{E}, 600 \mathrm{~m}$, vegetation near route, $1 \mathrm{0}, 2$ 우, 19.vi.2017; Zlaté Hory $1.7 \mathrm{~km} \mathrm{E,} 50^{\circ} 15^{\prime} 20^{\prime \prime} \mathrm{N}, 17^{\circ} 24^{\prime} 38^{\prime \prime} \mathrm{E}, 835 \mathrm{~m}$, forest underbrush mixed forest, 1 ๙ , 1 ㅇ,

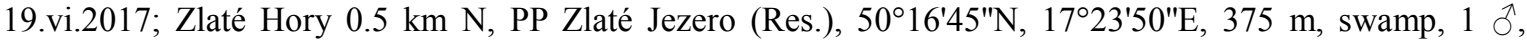

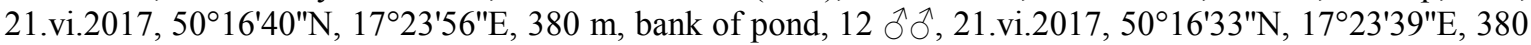
$\mathrm{m}$, meadow near stream Zlatý potok, $90^{\lambda} \mathrm{o}^{\prime}, 1$ ㅇ, 21.vi.2017, $50^{\circ} 16^{\prime} 44^{\prime \prime} \mathrm{N}, 7^{\circ} 23^{\prime} 40^{\prime \prime} \mathrm{E}, 380 \mathrm{~m}$, meadow near pond, $1 \mathrm{O}^{\star}$, 21.vi.2017; Zlaté Hory $1.5 \mathrm{~km} \mathrm{SW}$, „Zlatorudné mlýny“, $50^{\circ} 15^{\prime} 02^{\prime \prime} \mathrm{N}, 17^{\circ} 21^{\prime} 50^{\prime \prime} \mathrm{E}, 460 \mathrm{~m}$, near stream Olešnice, 1 + , 20.vi.2017.

Chromatomyia fuscula (Zetterstedt, 1838)

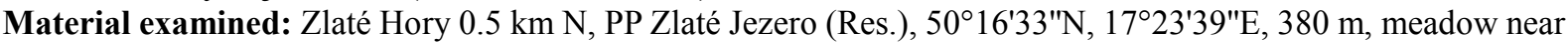
stream Zlatý potok, 1 đ, 21.vi.2017.

Chromatomyia milii (Kaltenbach, 1864)

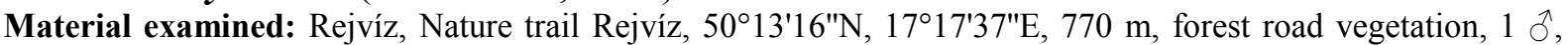
1 , 20.vi.2017, „U pomníku“, $50^{\circ} 13^{\prime} 35^{\prime \prime} \mathrm{N}, 17^{\circ} 17^{\prime} 46^{\prime \prime} \mathrm{E}, 800 \mathrm{~m}$, forest road vegetation, 2 ¿ $^{\lambda}$, 20.vi.2017;

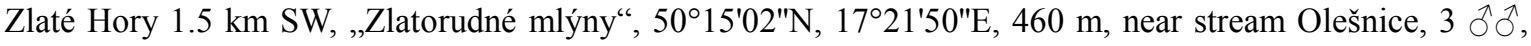
20.vi.2017.

Galiomyza galiivora (Spencer, 1969)

Material examined: Zlaté Hory $0.5 \mathrm{~km} \mathrm{E}, 50^{\circ} 15^{\prime} 30^{\prime \prime} \mathrm{N}, 17^{\circ} 24^{\prime} 11^{\prime \prime} \mathrm{E}, 520 \mathrm{~m}$, forest underbrush near forest road,

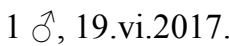

Remarks: Galiomyza galiivora is a Holarctic species (Papp \& Černý 2016); in Europe there are scattered records from Belgium, Corsica, Czech Republic, Germany, Greece, Hungary, Italy, Lithuania, Norway (Winqvist et al. 2020), Poland, Russia (Černý et al. 2021), Slovakia and Switzerland. In the Czech Republic it was first reported from the Pálava Biosphere Reserve, from NPR Kotel and Lednice (Černý \& Vála 1999) and later confirmed from further localities in Bohemia (Černý 2009, Černý et al. 2001, 2013; Černý \& Heřman 2015) and southern Moravia (Černý 2001a, b). The above record is the first from the Northern Moravia and Silesia. 
Liriomyza europaea Zlobin, 2003

Material examined: Rejvíz, Nature trail Rejvíz, $50^{\circ} 13^{\prime} 16^{\prime \prime} \mathrm{N}, 17^{\circ} 17^{\prime} 37^{\prime \prime} \mathrm{E}, 770 \mathrm{~m}$, forest road vegetation, $1 \mathrm{O}^{7}$, 20.vi.2017; Zlaté Hory 2 km E, „Biskupská kupa“, 50²15'24"N, 17²4'43"E, 890 m, vegetation near viewtower, 1 o, 19.vi.2017.

Remarks: A European species originally described from Russia (European part), England and Spain. Subsequently, it has been found also in other countries of Europe: Austria, Czech Republic, Finland, Germany Greece, Netherlands, Sweden and Switzerland (Papp \& Černý 2017, Černý \& Bächli 2018, Černý et al. 2021, von Tschirnhaus \& van Wielink 2020). In the Czech Republic it was first found in southern Moravia, in the Podyjí NP (locality Havraníky, see Černý et al. 2006). In Bohemia it is known from Úpská jáma in the Krkonoše Mts (Černý et al. 2009) and also from a hitherto unpublished record from southern Bohemia: CHKO Třeboňsko, Suchdol nad Lužnicí, Hrdlořezy 1 km NW, 4852'26"N, 1451'13"E, 470 m, forest, 1 Oૈ, 25.v.2015, M. Černý leg. and coll. The records listed above are the first from the Northern Moravia and Silesia.

Liriomyza flaveola (Fallén, 1823)

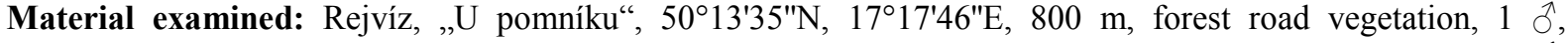
20.vi.2017; Zlaté Hory $0.5 \mathrm{~km} \mathrm{E}, 50^{\circ} 15^{\prime} 30^{\prime \prime} \mathrm{N}, 17^{\circ} 24^{\prime} 11^{\prime \prime E}, 520 \mathrm{~m}$, forest underbrush near forest road, 1 ,

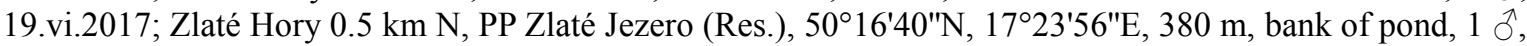

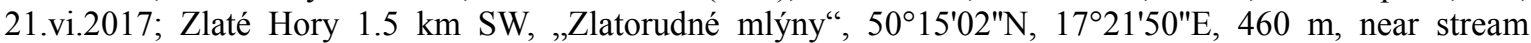
Olešnice, 1 ô, 20.vi.2017.

Liriomyza phryne Hendel, 1931

Material examined: Zlaté Hory $0.5 \mathrm{~km}$ N, PP Zlaté jezero (Res.), 50¹6'45"N, 17²3'50"E, 375 m, swamp, 1 ○, 21.vi.2017.

Liriomyza ptarmicae de Meijere, 1925

Material examined: Zlaté Hory $0.5 \mathrm{~km} \mathrm{~N}$, PP Zlaté jezero (Res.), 50 $16^{\prime} 32^{\prime \prime} \mathrm{N}, 17^{\circ} 23^{\prime} 43^{\prime \prime E}, 375 \mathrm{~m}$, meadow near stream Zlatý potok, 1 Ô, 21.vi.2017.

Liriomyza puella (Meigen, 1830)

Material examined: Zlaté Hory $1.5 \mathrm{~km} \mathrm{~S}$, „Zlatorudné mlýny“, $50^{\circ} 15^{\prime} 02^{\prime \prime} \mathrm{N}, 17^{\circ} 21^{\prime} 50^{\prime \prime} \mathrm{E}, 460 \mathrm{~m}$, near stream Olešnice, 1 ô, 20.vi.2017.

Remarks: A species only known from the W. Palaearctic (Papp \& Černý 2017). In the Czech Republic, there are records of L. puella from Moravia from Troubsko and Zvěrkovice (Černý \& Vlk 2001). The species has later been confirmed from Bohemia in the Jizerské hory Mts (Bílý potok pod Smrkem and PR Meandry Smědé, see Černý 2009). The above record is the first from the Northern Moravia and Silesia.

\section{Liriomyza pusilla (Meigen, 1830)}

Material examined: Zlaté Hory $2 \mathrm{~km}$ E, „Biskupská kupa“, 50¹5'24"N, 17²4'43"E, 890 m, vegetation near view-tower, 1 ô, 19.vi.2017.

Liriomyza soror Hendel, 1931

Material examined: Zlaté Hory $0.5 \mathrm{~km} \mathrm{~N}$, PP Zlaté jezero (Res.), 50 $16^{\prime} 32^{\prime \prime} \mathrm{N}, 17^{\circ} 23^{\prime} 43^{\prime \prime} \mathrm{E}, 375 \mathrm{~m}$, meadow near

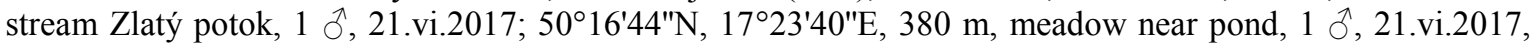
$50^{\circ} 16^{\prime} 45^{\prime \prime} \mathrm{N}, 17^{\circ} 23^{\prime} 47^{\prime \prime} \mathrm{E}, 380 \mathrm{~m}$, bank of pond, 1 oे, 1 , 21.vi.2017.

Metopomyza flavonotata (Haliday, 1833)

Material examined: Zlaté Hory $0.5 \mathrm{~km} \mathrm{E}, 50^{\circ} 15^{\prime} 32^{\prime \prime} \mathrm{N}, 17^{\circ} 24^{\prime} 16^{\prime \prime} \mathrm{E}, 530 \mathrm{~m}$, forest underbrush near forest road,

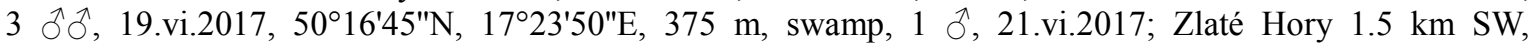
„Zlatorudné mlýny“, 50¹5'02"N, 17²21'50"E, 460 m, near stream Olešnice, 1 + , 20.vi.2017.

Metopomyza xanthaspioides (Frey, 1946)

Material examined: Zlaté Hory $0.5 \mathrm{~km}$ N, PP Zlaté Jezero (Res.), 50¹6'33"N, 17²3'39"E, $380 \mathrm{~m}$, meadow near stream Zlatý potok, 1 §, 3 우, 21.vi.2017.

Phytoliriomyza melampyga (Loew, 1869)

Material examined: Zlaté Hory $0.5 \mathrm{~km} \mathrm{E}, 50^{\circ} 15^{\prime} 35^{\prime \prime} \mathrm{N}, 17^{\circ} 24^{\prime} 03^{\prime \prime} \mathrm{E}, 475 \mathrm{~m}$, forest underbrush near forest road, 1 ô, 1 + , 19.vi.2017.

Phytomyza aurei Hering, 1931

Material examined: Zlaté Hory $1.5 \mathrm{~km} \mathrm{SW}$, „Zlatorudné mlýny“, 50¹5'02"N, 17²1'50"E, $460 \mathrm{~m}$, near stream Olešnice, 1 ð̊, 20.vi.2017; Zlaté Hory 2 km E, „Biskupská kupa“, 50¹5'24"N, 17²4'43"E, 890 m, vegetation near view-tower, 1 đ, 19.vi.2017.

Remarks: This West Palaearctic species has been recorded from the Czech Republic, Germany, Hungary, Lithuania, Poland, Slovakia, Switzerland, and Turkey (Papp \& Černý 2020), and recently also from Ukraine (Guglya 2021). In the Check list of Czechoslovak Insects II (Vála 1987) this species is listed as new for Moravia 
but without faunistic data of specimens examined. First valid data are from CHKO Bílé Karpaty, from Hutě (Černý 2001b) and PP Jalovcová louka nr. Trnava u Zlína (Černý 2001a). In Bohemia it was first confirmed from the Šumava Mts (Vimperk, cf. Černý \& Vlk 2001). Subsequently it has been collected in the Jizerské hory Mts in Rejdice (Černý 2009) and later also in NPR Karlštejn in Kubrychtova bouda (Černý \& Heřman 2015). The above records are the first from Northern Moravia and Silesia.

Phytomyza chaerophylli Kaltenbach, 1856

Material examined: Zlaté Hory $0.5 \mathrm{~km}$ E, $50^{\circ} 15^{\prime} 32^{\prime \prime} \mathrm{N}, 17^{\circ} 24^{\prime} 16^{\prime \prime} \mathrm{E}, 530 \mathrm{~m}$, forest underbrush near forest road, $1 \lesssim, 19 . v i .2017$.

Phytomyza cirsii Hendel, 1923

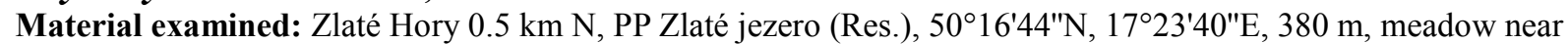
pond, 1 गे, 21.vi.2017.

Remarks: A W. Palaearctic species, largely recorded from central, western and northern Europe and also known from Turkey (Papp \& Černý 2020). In the Czech Republic it was first confirmed in the Pálava Biosphere Reserve (locality Milovice, see Černý \& Vála 1999); later also encountered in Bohemia (Černý 2009, Černý et al. 2001, 2013) and in southern Moravia in the CHKO Bílé Karpaty (NPR Čertoryje-Vojšické louky, cf. Černý 2001b). The above record is the first from Northern Moravia and Silesia.

Phytomyza crassiseta Zetterstedt, 1860

Material examined: Zlaté Hory 2 km E, „Biskupská kupa“, 50¹5'24"N, 17²4'43"E, 890 m, vegetation near view-tower, 1 ô, 19.vi.2017.

Phytomyza evanescens Hendel, 1920

Material examined: Zlaté Hory $1.5 \mathrm{~km} \mathrm{SW}$, „Zlatorudné mlýny“, 50¹5'02"N, $17^{\circ} 21^{\prime} 50^{\prime \prime E}, 460 \mathrm{~m}$, near stream Olešnice, 1 ふૈ, 20.vi.2017.

Phytomyza plantaginis Robineau-Desvoidy, 1851

Material examined: Zlaté Hory 0.8 km E, 50¹5'30"N, 17²4'35"E, 555 m, meadow near forest, 1 ô, 19.vi.2017.

Phytomyza podagrariae Hering, 1954

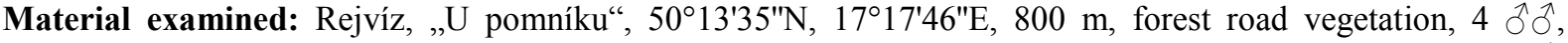
20.vi.2017; Zlaté Hory $0.5 \mathrm{~km} \mathrm{E}, 50^{\circ} 15^{\prime} 32^{\prime \prime} \mathrm{N}, 1^{\circ} 24^{\prime} 16^{\prime \prime} \mathrm{E}, 530 \mathrm{~m}$, forest underbrush near forest road, $1 \overbrace{}^{\wedge}$, 19.vi.2017, 50 $15^{\prime} 35^{\prime \prime} \mathrm{N}, 17^{\circ} 24^{\prime} 03^{\prime \prime} \mathrm{E}, 475 \mathrm{~m}$, forest underbrush near forest road, 1 ô, 19.vi.2017.

Phytomyza ranunculi (Schrank, 1803)

Material examined: Zlaté Hory 0.5 km N, PP Zlaté Jezero (Res.), 50¹6'33"N, 17²3'39"E, 380 m, meadow near stream Zlatý potok, 1 ㅇ, 21.vi.2017.

Phytomyza robustella Hendel, 1936

Material examined: Zlaté Hory 1.5 km SW, „Zlatorudné mlýny“, 50¹5'02"N, 17²21'50"E, 460 m, near stream Olešnice, 1 Oे, 20.vi.2017.

Phytomyza vilnensis Pakalniškis, 1998

Material examined: Zlaté Hory $2 \mathrm{~km}$ E, „Biskupská kupa“, 50¹5'24"N, 17²4'43"E, 890 m, vegetation near view-tower, 2 đo 19. .vi.2017.

Remarks: A European species with a poorly known distribution. It was described from Lithuania and later recorded from Hungary (Papp \& Černý 2020) and Slovakia (Černý \& Roháček 2020). It has been reported only once from the Czech Republic, viz. from PP Zlaté Jezero (Res.) near Zlaté Hory (Černý et al. 2021). The record listed above is additional to the occurrence of in the vicinity of the Zlaté Hory.

Pseudonapomyza atra (Meigen, 1830)

Material examined: Zlaté Hory $0.5 \mathrm{~km} \mathrm{~N}$, PP Zlaté Jezero (Res.), 50 $16^{\prime} 33^{\prime \prime} \mathrm{N}, 17^{\circ} 23^{\prime} 39^{\prime \prime} \mathrm{E}, 380 \mathrm{~m}$, meadow near

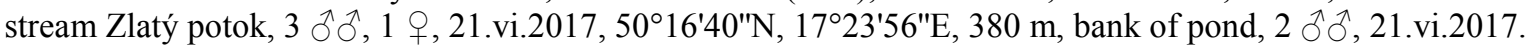

Pseudonapomyza palliditarsis Černý, 1992

Material examined: Zlaté Hory 0.5 km N, PP Zlaté Jezero (Res.), 50¹6'33"N, 17²3'39"E, 380 m, meadow near stream Zlatý potok, 1 ㅇ, 21.vi.2017.

Remarks: An insufficiently known species, probably Palaearctic in distribution, originally described from Zvěrkovice (distr. Třebíč, southern Moravia, Czech Republic) (Černý 1992) and subsequently recorded from Bulgaria, Hungary, Poland, European Russia, Slovakia, Spain, Ukraine, Uzbekistan and from Yakutia (Russian E. Siberia) (Papp \& Černý 2017). In the Czech Republic, other than the type locality, it is known from the Pálava Biosphere Reserve (Lednice, NPR Lednické rybníky and Podivín, see Černý \& Vála 1999), Podyjí NP (Černý et al. 2006), Milotice nad Bečvou (Černý 2019) and from several localities in Bohemia (PP Stroupeč and NPR Úhošt', see Černý 2013). The above record is the first from Northern Moravia and Silesia. 


\section{Discussion and conclusions}

Although the faunal investigation in the vicinity of the Zlaté Hory town was realized within a short time in June 2017, its results have demostrated the occurrence of a relatively rich speciesspectrum of the family Agromyzidae. This territory has hitherto been largely neglected by dipterists and, consequently, the faunal data obtained are largely new for the area. Altogether 43 species of Agromyzidae have been recorded, including 36 species found in the cadastre of Zlaté Hory for the first time and 29 species which are new additions to the fauna of Czech Silesia: Agromyza conjuncta, A. mobilis, A. seticercus, Amauromyza (Cephalomyza) monfalconensis, Cerodontha (Poemyza) beigerae, Cerodontha (Xenophytomyza) biseta, Galiomyza galiivora, Liriomyza europaea, L. ptarmicae, L. puella, L. pusilla, L. soror, Melanagromyza aenea, M. sativae, Metopomyza flavonotata, Met.xanthaspioides, Ophiomyia orbiculata, O. pulicaria, Phytoliriomyza melampyga, Phytomyza aurei, Ph. cirsii, Ph. crassiseta, Ph. evanescens, Ph. chaerophylli, Ph. plantaginis, Ph. podagrariae, Ph. robustella, Pseudonapomyza atra and Ps. palliditarsis. The nine faunistically most interesting species, viz. Agromyza seticercus, Cerodontha (Poemyza) beigerae, Galiomyza galiivora, Liriomyza europaea, L. puella, Phytomyza aurei, Ph. cirsii, $P h$. vilnensis and Pseudonapomyza palliditarsis, are provided with comments on their general distribution and occurrence in the Czech Republic. There is only one previous paper devoted to Agromyzidae in Silesia and containing data from the Zlaté Hory cadastre, viz. Vála \& Roháček (1983). In the latter study 21 species were reported from the peat-bog at Rejvíz, namelyAgromyza cinerascens Macquart, 1835; A. albipennis Meigen, 1830; A. nigripes Meigen, 1830; Cerodontha (Butomomyza) scirpi (Karl, 1926); C. (Cerodontha) denticornis (Panzer, 1806); C. (Dizygomyza) morosa (Meigen, 1830); C. (D.) suturalis (Hendel, 1931); C. (Icteromyza) churchillensis Spencer, 1969; C. (Poemyza) superciliosa (Zetterstedt, 1860); C. (P.) incisa (Meigen, 1830); Chromatomyia fuscula (Zetterstedt, 1838); Ch. nigra (Meigen, 1830); Liriomyza flaveola (Fallén, 1823); L. phryne Hendel, 1931; L. virgo (Zetterstedt, 1838); Metopomyza interfrontalis (Melander, 1913); Napomyza scrophulariae Spencer, 1966; Phytomyza flavofemorata Strobl, 1893; Ph. ranunculi (Schrank, 1803); Ph. rufipes Meigen, 1830 and Ph. spinaciae Hendel, 1935.

Acknowlegment: I would like to thank Jindřich Roháček (Opava, Czech Republic) for valuable comments and assistance with the early draft of the manuscript and David Gibbs (Weston-super-Mare, Great Britain) for the language revision and other improvement of the manuscript.

\section{References}

Černý M. (1992): A revision of Czechoslovak species of Pseudonapomyza Hendel, with description of four new species (Diptera, Agromyzidae). - Acta Entomologica Bohemoslovaca 89: 451-465.

- (2001a): Agromyzidae (Diptera) of the Zlín district (Czech Republic). In: Chvála M. (ed.): Dipterologica bohemoslovaca 10. - Acta Universitatis Carolinae-Biologica 45 (2001): 31-40.

- (2001b): Př́ispěvek k poznání Agromyzidae (Diptera) Chráněné krajinné oblasti Bílé Karpaty. A contribution to the knowledge of Agromyzidae (Diptera) of the Bílé Karpaty Protected Landscape Area. - Sborník přírodovědného klubu v Uherském Hradišti 6: 244-258.

- (2009): Vrtalkovití (Diptera: Agromyzidae) Jizerských hor a Frýdlantska. Agromyzidae (Diptera) of the Jizerské hory Mts and Frýdlant region (Northern Bohemia, Czech Republic). - Sborník Severočeského Muzea Př́rodní Vědy, Liberec 27: 115-140.

- (2013): Additional records of Agromyzidae (Diptera) from the West Palaearctic Region. - Časopis Slezského Muzea Opava (A) 62: 281-288.

- (2018): Additional new records of Agromyzidae (Diptera) from the Palaearctic Region. - Acta Musei Silesiae, Scientiae Naturales 67: 117-137.

- (2019): Př́ispěvek k faunistice minujících much vrtalkovitých (Agromyzidae, Diptera) z oblasti okolo Bečvy u Skaličky a Milotic nad Bečvou (střední Morava, Česká republika). A contribution to the faunistics of mining Agromyzidae (Diptera) from the Bečva River area near the villages of Skalička and Milotice nad Bečvou (central Moravia, Czech Republic). - Acta Carpathica Occidentalis 9 (2018): 33-52.

Černý M., Barták M. \& Kubík Š. (2006): Agromyzidae. Pp. 285-300. In: Barták M. \& Kubík Š. (eds): Diptera of Podyjí National Park and its Environs. Česká zemědělská univerzita v Praze, 432 pp.

- (2013): Agromyzidae (Diptera) of Vráž nr. Písek (Czech Republic). Pp. 111-130. In: Kubík Š. \& Barták M. (eds): Workshop on biodiversity, Jevany. Česká zemědělská univerzita v Praze, 436 pp. Printed version (ISBN 978-80-213-2423-7) + CD (ISBN 978-80-213-2424-4). 
Černý M., Barták M. \& Vaněk J. (2009): Vrtalkovití (Diptera, Agromyzidae) vysokých poloh Krkonoš. Agromyzidae (Diptera) of high altitudes of the Krkonoše Mts. - Opera Corcontica 46: 185-197.

Černý M. \& Bächli G. (2018): New records of Agromyzidae (Diptera) from Switzerland and an updated checklist. - Alpine Entomology 2: 115-137.

Černý M. \& Heřman P. (2015): Vrtalkovití (Diptera: Agromyzidae) Českého krasu (Leaf-miner flies (Diptera: Agromyzidae) of the Bohemian Karst (central Bohemia, Czech Republic)). - Západočeské entomologické listy 6: 50-65. Online: http://www.zpcse.cz/entolisty/entolisty.html, 12-10-2015.

Černý M. \& Roháček J. (2020): New records of the Agromyzidae (Diptera) from Muránska planina National Park (Central Slovakia). - Acta Musei Silesiae, Scientiae Naturales 69: 97-140.

Černý M., von Tschirnhaus M. \& Winqvist K. (2021): First records of Palaearctic Agromyzidae (Diptera) from 40 countries and major islands - Acta Musei Silesiae, Scientiae Naturales 69: 193-229.

Černý M. \& Vála M. (1999): Agromyzidae In: Rozkošný R. \& Vaňhara J. (eds): Diptera of the Pálava Biosphere Reserve of UNESCO, II. - Folia Facultatis Scientiarum Naturalium Universitatis Masarykianae Brunensis, Biologia 100: 297-310.

- (2005): Faunistic records from the Czech and Slovak Republics: Diptera: Agromyzidae. In: Kubík Š. \& Barták M. (eds): Dipterologica bohemoslovaca 11. - Folia Facultatis Scientiarum Naturalium Universitatis Masarykianae Brunensis, Biologia 109 (2004): 335-343.

Černý M., Vála M. \& Barták M. (2001): Agromyzidae. Pp. 349-364. In: Barták M. \& Vaňhara J. (eds): Diptera in an Industrially Affected Region (North-Western Bohemia, Bílina and Duchcov Environs), II. - Folia Facultatis Scientiarum Naturalium Universitatis Masarykianae Brunensis, Biologia 105: 241-514.

Černý M. \& V1k R. (2001): Faunistic records. Agromyzidae. In: Chvála M. (ed.): Dipterologica bohemoslovaca 10. - Acta Universitatis Carolinae-Biologica 45 (2001): 193-197.

Guglya Y.A. (2016): A study of the fauna of leaf-miner flies of the subfamily Agromyzinae (Diptera: Agromyzidae) of Ukraine. Report 4. Thirteen new species for the fauna of Ukraine. - The Kharkov Entomol. Soc. Gaz. 24 (2): 17-24.

- (2021): Rearing mining flies (Diptera: Agromyzidae) from host plants as an instrument for associating females with males, with the description of seven new species. - Zootaxa 5014 (1): 1-158. https://www.mapress. $\mathrm{com} / \mathrm{j} / \mathrm{zt}$

Heřman P., Preisler J., Černý M., Dvořák L., Chvála M. \& Máca J. (2019): Nálezy dvoukřídlých vybraných čeledí (Diptera) na území CHKO Brdy v období 2005-2017. Data on the selected Diptera families from the Brdy Highlands Protected Landscape Area (Central Bohemia, the Czech Republic) in 2005-2017. - Bohemia centralis, Praha 35: 525-540.

Martinez M. (2013) Fauna Europaea: Agromyzidae. In: Beuk P. \& Pape T. (eds): Fauna Europaea: Diptera, Flies. Fauna Europaea version 2017.06. http://fauna-eu.org. Accessed 20 October 2021.

Nowakowski T.J. (1973): Monographie der europäischen Arten der Gattung Cerodontha Rond. - Annales zoologici 31(1): 1-327.

Papp L. \& Černý M. (2015): Agromyzidae (Diptera) of Hungary. Volume 1. Agromyzinae. Pars Ltd, Nagykovácsi, Hungary 2015: 416 pp.

- (2016): Agromyzidae (Diptera) of Hungary. Volume 2. Phytomyzinae I. Pars Ltd, Nagykovácsi, Hungary 2016 : $385 \mathrm{pp}$.

- (2017): Agromyzidae (Diptera) of Hungary. Volume 3. Phytomyzinae II. Pars Ltd, Nagykovácsi, Hungary 2017 : $427 \mathrm{pp}$.

- (2020): Agromyzidae (Diptera) of Hungary. Volume 4. Phytomyzinae III. Pars Ltd, Nagykovácsi, Hungary 2019 : $708 \mathrm{pp}$.

Spencer K.A. (1990): Host Specialization in the World Agromyzidae (Diptera). Series Entomologica 45: 1-444.

Starý B. (1930): O minujícím hmyzu v zemi Moravskoslezské. - Acta Societatis Scientiarum Naturalium Moravicae 6(6): 1-118.

Vála M. (1987): Agromyzidae. Pp. 229-233. In: Ježek J. (ed.): Check list enumeratio insectorum Bohemoslovakiae II (Diptera). - Acta faunistica entomologica Musei Nationalis Pragae 18: 341 pp.

Vála M. \& Roháček J. (1983): Diptera Acalyptrata rašeliništ' severní Moravy (ČSSR). Č́st 5. Agromyzidae. Acalypterate Diptera of peat-bogs in North Moravia (Czechoslovakia). Part 5. Agromyzidae. - Čas. Slez. Muz. Opava (A) 32: 193-214.

von Tschirnhaus M. \& van Wielink P. (2020): Agromyzidae. Pp. 361. In: van Wielink P., Felix R., van Kemenade J., Mol A., Peeters T. \& Stooker G. (eds): De Kaaistoep, het best onderzochte stuk natuur in Nederland [De Kaaistoep, the best studied nature area in the Netherlands]. Knnv-afd. Tilburg, 720 pp.

Winqvist K., Černý M. \& Andersen T. (2020): Twenty species of Agromyzidae (Diptera) from Hedmark and Finnmark not previously recorded from Norway. - Norwegian Journal of Entomology 69: 125-131.

Zavřel H. (1953): Minující hmyz na Kotouči a v okolí Štramberka I. Mining insects on Kotouč Mt. and in the environs of Štramberk I. - Př́rodovědecký sborník Ostravského kraje 14: 416-422, 3 refs. 


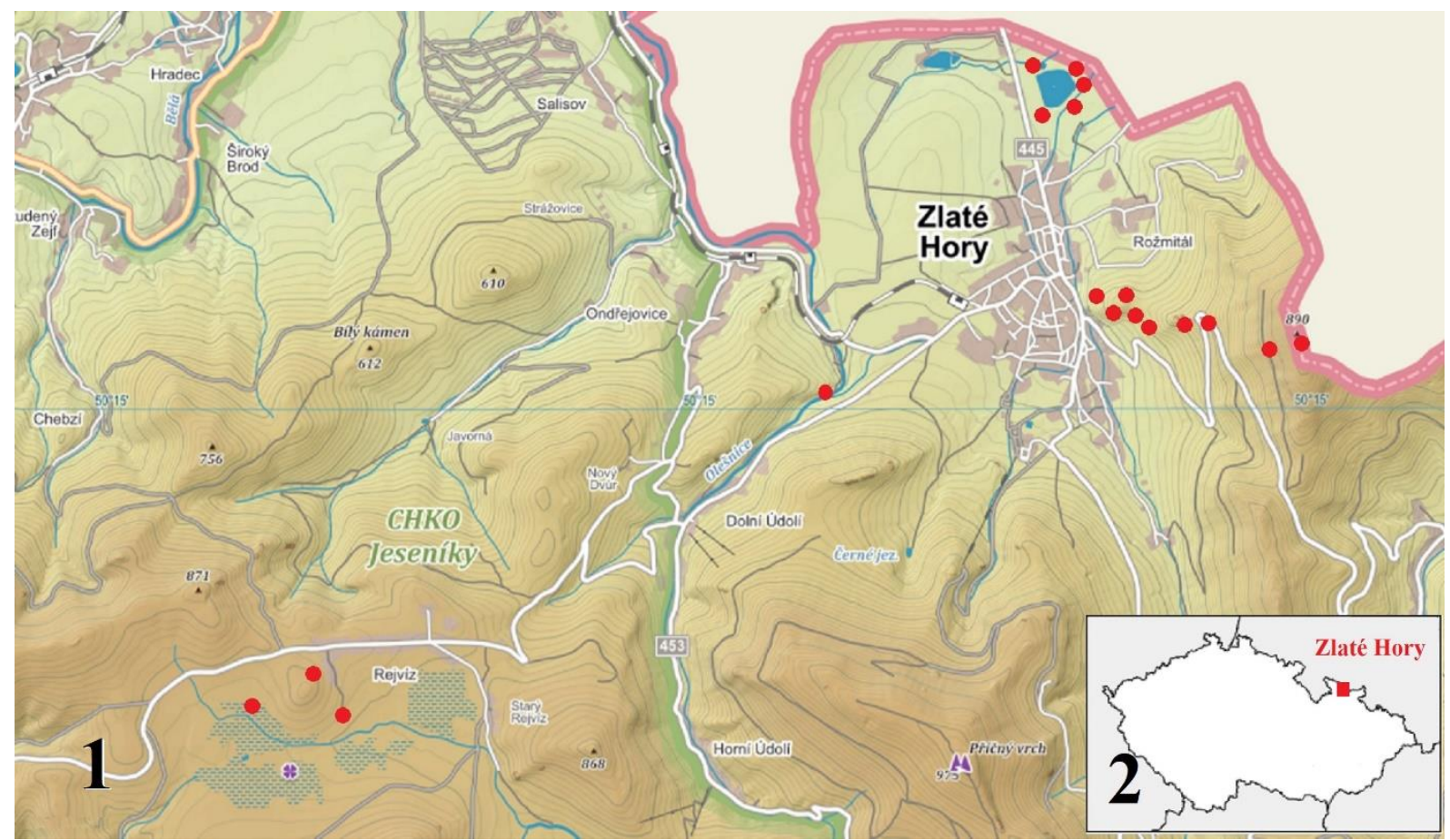

Figs 1-2: Maps. 1 - localities visited in the cadastre of the Zlaté Hory town (red circles); 2 - situation of the cadastre of the Zlaté Hory town in the Czech Republic.

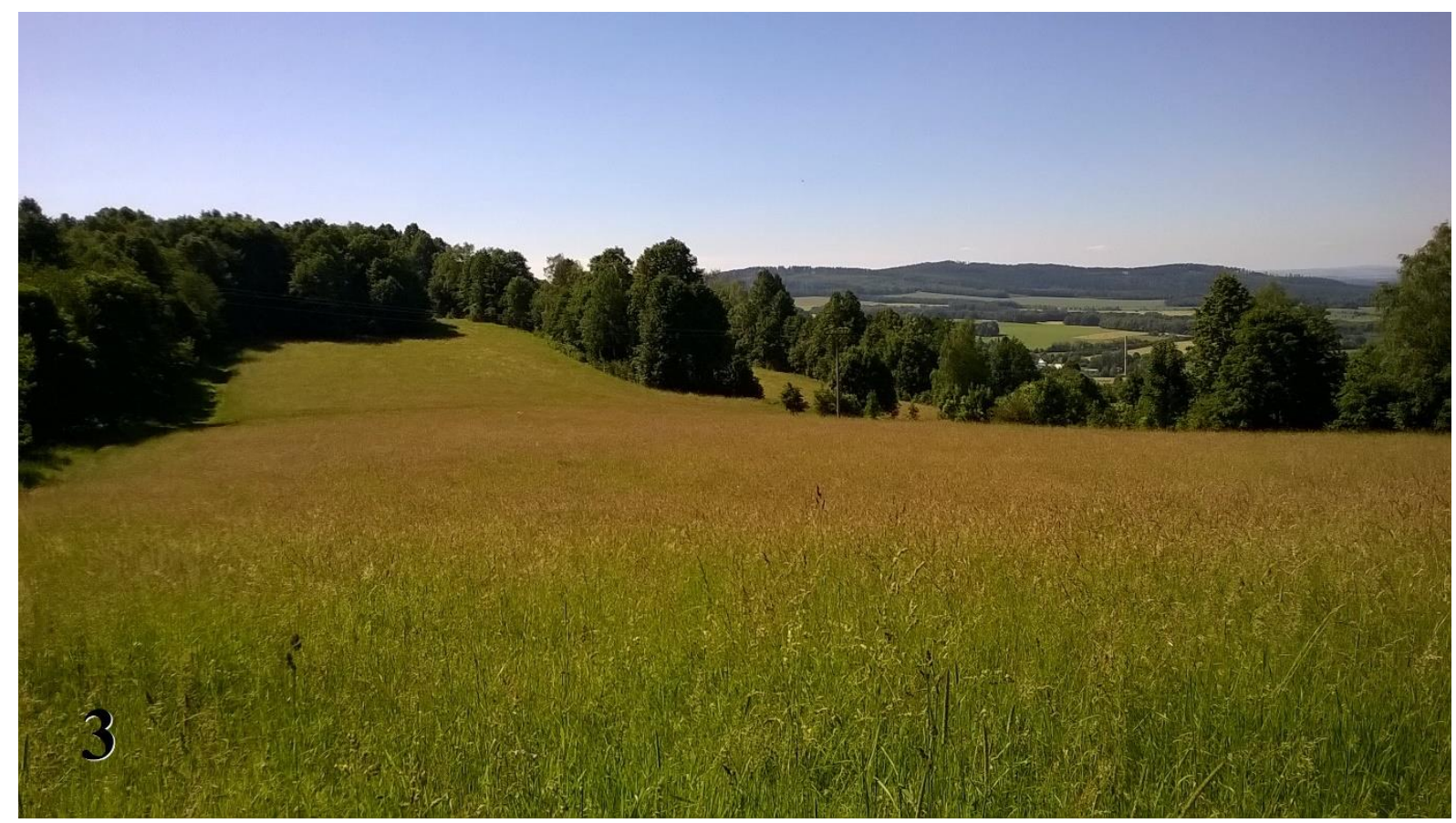

Fig 3: Zlaté Hory $0.8 \mathrm{~km}$ E, meadow near forest, habitat of Amauromyza (Cephalomyza) monfalconensis (Strobl). Photo by M. Černý. 


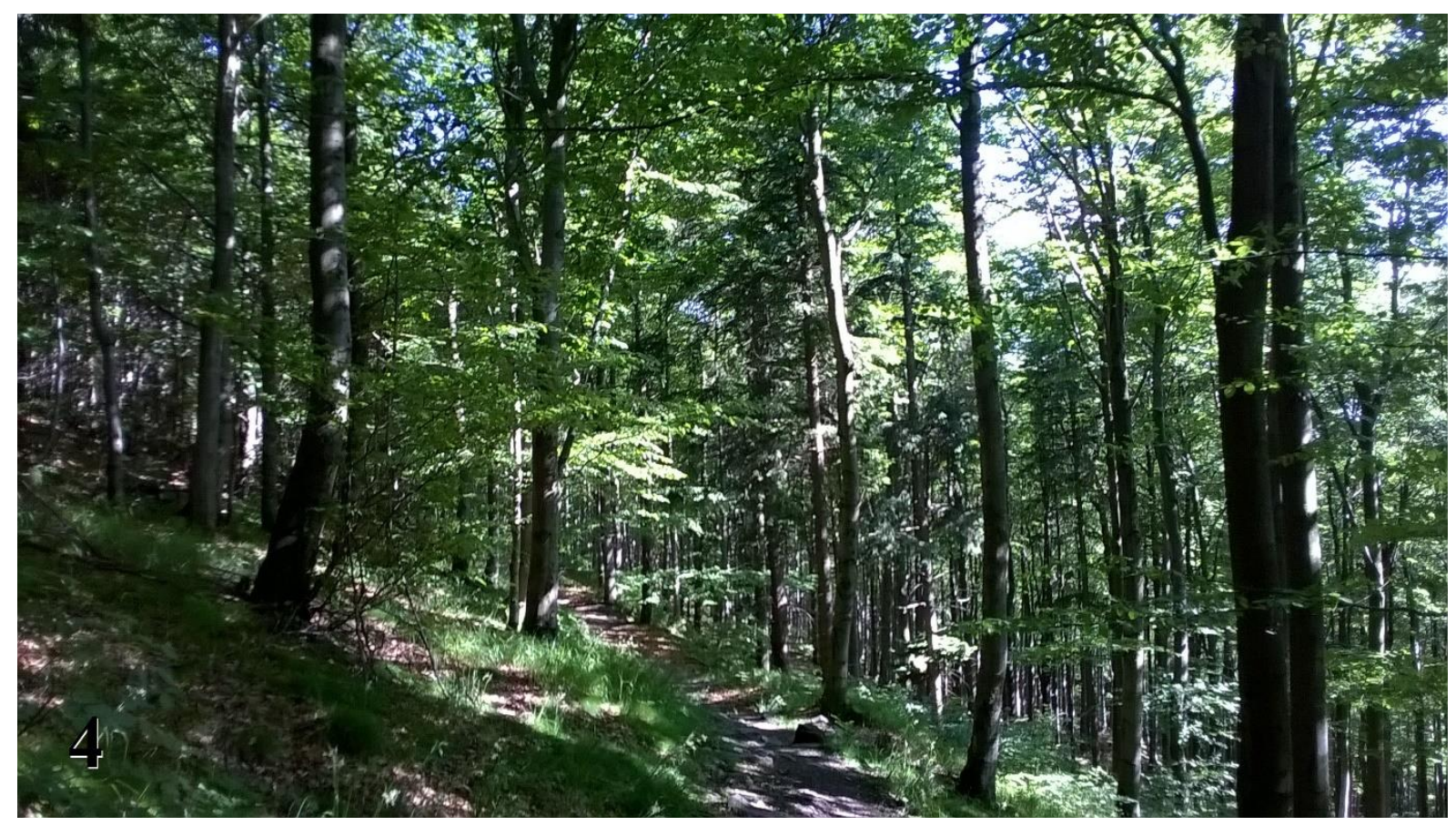

Fig 4: Zlaté Hory $2 \mathrm{~km}$ E, mixed (largely beech) forest with rich undergrowthin the vicinity of lookout tower „Biskupská kupa“. Habitat of Liriomyza europaea Zlobin and Phytomyza vilnensis Pakalniškis. Photo M. Černý.

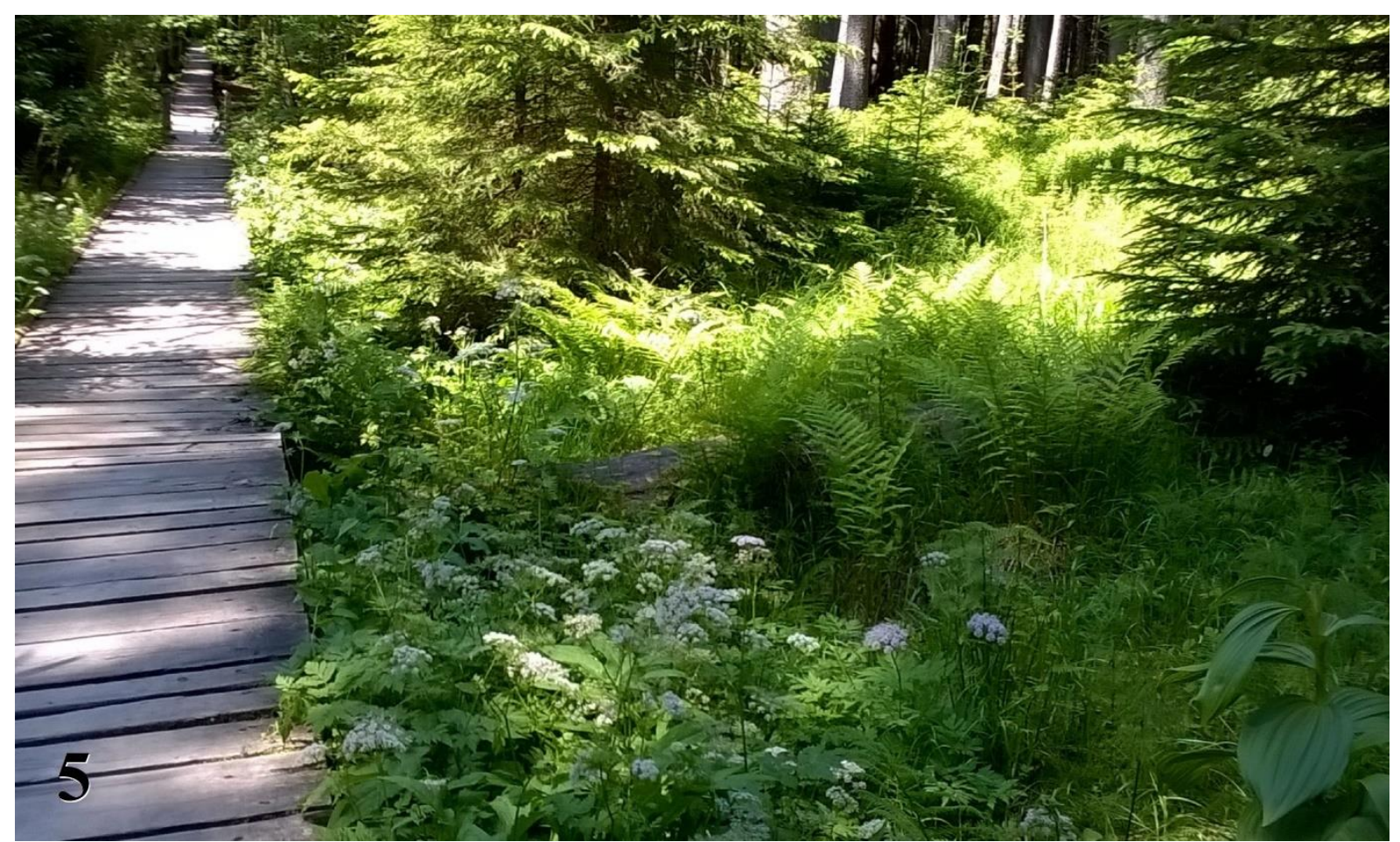

Fig 5: NPR Rejvíz, Nature trail Rejvíz, undergrowth of wet spruce forest along trail. Habitat of Cerodontha (Poemyza) beigerae Nowakowski. Photo by M. Černý. 


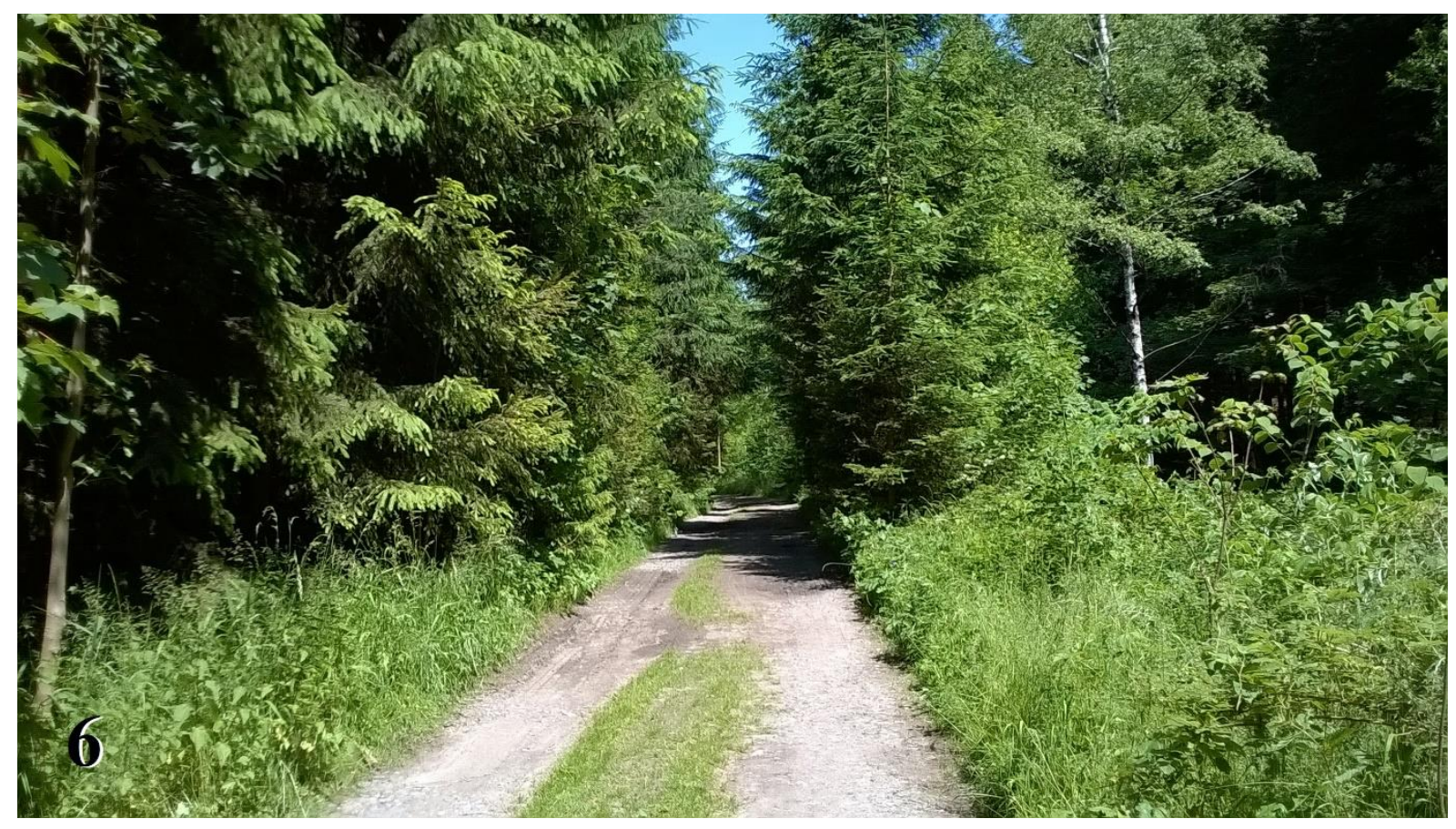

Fig 6: Zlaté Hory $1.5 \mathrm{~km} \mathrm{SW}$, Zlatorudné doly, woodland road alog river Olešnice. Habitat of Liriomyza puella (Meigen) and Phytomyza aurei Hering. Photo by M. Černý.

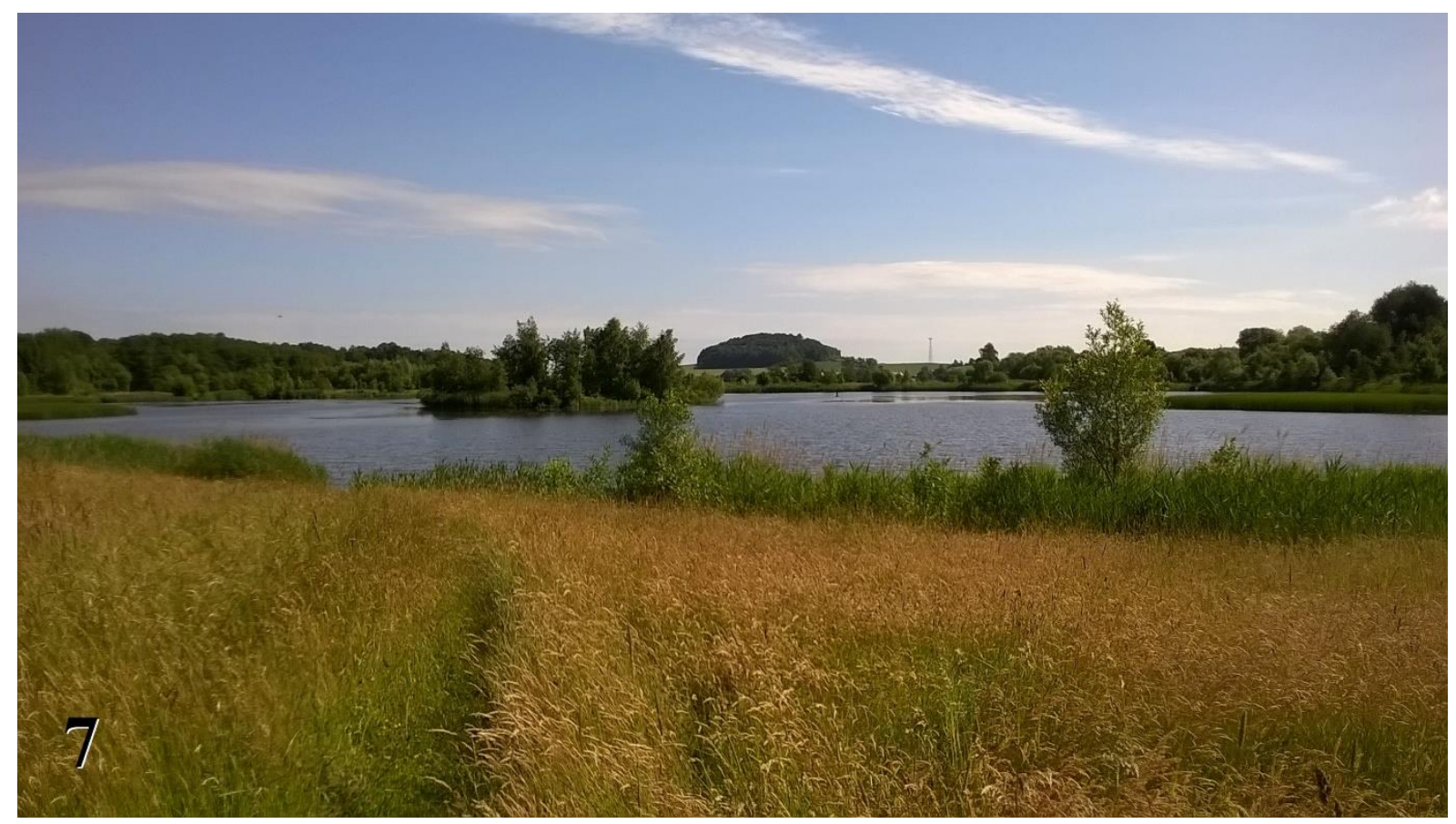

Fig 7: Zlaté Hory $0.5 \mathrm{~km} \mathrm{~N}$, Zlaté jezero pond, meadows and marshland habitats at shores of water reservoir. Photo by M. Černý.

Author's address: Miloš Černý, CZ-763 63 Halenkovice 1, Czech Republic.

E-mail: cerny.milos@centrum.cz 